\section{rev Psi}

Revista de Psicología (UNLP)

https://revistas.unlp.edu.ar/revpsi

\title{
La ansiedad en la población argentina en el contexto de pandemia por el COVID-19
}

\author{
Marcelo Ceberio ${ }^{1,2} \quad$ Gilda Mariel Jones ${ }^{1,2} \quad$ María Gabriela Benedicto ${ }^{1,2}$
}

Correspondencia

mariel_psico@yahoo.com.ar

Filiaciones institucionales

${ }^{1}$ LINCS- Laboratorio de Investigación en $\quad{ }^{2}$ Escuela Sistémica Argentina (Argentina)

Neurociencias y Ciencias Sociales (Universidad de

Flores) (Argentina)

\section{Resumen}

El artículo investiga los niveles de ansiedad que pudieron desencadenarse en la población de Argentina en relación con la situación crítica de la aparición del COVID-19. Se tiene en cuen-ta que la ansiedad es una emoción absolutamente esperable en este tipo de contexto actual, tan desestructurante. Pero no es solo la pandemia, sino también la reclusión mediante una cua-rentena obligatoria, que hace presente la incertidumbre. Pandemia, aislamiento e incertidum-bre activan una tríada emocional como el miedo, la ansiedad y la angustia. En este período, cumplidos los primeros 15 días de cuarentena, mediante el cuestionario STAI, se evaluó la ansiedad rasgo y estado. Notablemente, la investigación no arrojó los resultados esperados, con lo cual se articula una serie de hipótesis interpretativas en pos de entender el fenómeno. Se espera aplicar el mismo instrumento, pero a partir de los 75 días, para poder realizar una comparación.

Palabras clave

pandemia | ansiedad | COVID-19 | adaptación | resiliencia

Cómo citar

Ceberio, M., Jones, G. M. y Benedicto, G. (2021). La ansiedad en la población argentina en el contexto de pandemia por el COVID-19. Revista de Psicología, 20(2), 106-122. нтTPs://DX.DOI. ORG/10.24215/2422572XE081

\section{Proceso editorial}

Recibido

13 jul. 2020

Aceptado

2 feb. 2021
1 ra decisión

7 dic. 2020

Publicado 15 feb. 2021
ISSN

2422-572X

Licencia

Licencia de Cultura Libre CC-BY 4.0

(Compartir - Adaptar - Atribuir)

Entidad editora

RevPsi es una publicación de la

Facultad de Psicología (Universidad

Nacional de La Plata, Argentina) 


\section{Ansiedade na população argentina no contexto da pandemia da COVID-19}

\section{Resumo}

$\mathrm{O}$ artigo investiga os níveis de ansiedade que poderiam ser desencadeados na população argentina em relação à situação crítica do aparecimento do COVID-19. Levando em consideração que a ansiedade é uma emoção absolutamente esperada nesse tipo de contexto atual, tão destrutivo. Porém no é apenas a pandemia, mas também a reclusão através de uma quarentena obrigatória, que torna um presente dividoso. Pandemia, isolamento, incerteza, ativam uma tríade emocional como o medo, ansiedade e angústia. Nesse período, após os primeiros 15 dias de quarentena, utilizando o questionário STAI, foram avaliadas as características e a ansiedade do estado. Notavelmente, a pesquisa não produziu os resultados esperados, o que articula uma série de hipóteses interpretativas para entender o fenômeno. Espera-se que o mesmo instrumento seja aplicado, após 75 dias, para poder comparação.

\section{Palavras-chave}

pandemia | ansiedade | COVID-19 | adaptação | resiliência

\section{Anxiety in the Argentine population in the context of the CO- VID-19 pandemic}

\section{Abstract}

The article investigates the levels of anxiety that may have been triggered in the population of Argentina in relation to the critical situation caused by the appearance of COVID-19. Anxie-ty is an emotion that is to be expected in this type of context, which is so unstructured. But it is not only the pandemic, but also confinement through an obligatory quarantine, which makes the uncertainty present. Pandemic, isolation, and uncertainty activate an emotional triad such as fear, anxiety, and anguish. During this period, once the first 15 days of quaran-tine had passed, we evaluated the trait and state of anxiety by means of the STAI question-naire. Remarkably, the research did not yield the expected results, so a series of interpretative hypotheses were articulated to understand the phenomenon. The same instrument is expected to be applied, but after 75 days, to make a comparison.

\section{Keywords}

pandemic | anxiety | COVID-19 | adaptation | resiliency 


\section{Aspectos destacados del trabajo}

- La ansiedad estado y la ansiedad rasgo de la población argentina es baja.

- Las mujeres evidencian mayores niveles de ansiedad.

- Los profesionales de la salud evidencian mayores niveles de ansiedad.

- Se observa en los argentinos una gran adaptabilidad a las crisis.

En la presente investigación se pretendió evaluar la ansiedad en contexto de pandemia en Argentina, entendiendo que en esta situación de crisis -potencialmente traumática- la ansiedad puede ser la emoción frente a la incertidumbre. Esta inferencia se construye sobre la base de que este estímulo desestructurante pone a prueba los esquemas cognitivos y emocionales habituales de la persona, a la vez que cumple con las características del criterio A del Trastorno de Estrés Postraumático del DSM-IV: "evento que implica una amenaza de muerte o lesiones graves o una amenaza para la integridad física o lesiones graves del yo o de los otros" (APA, 1994). A esto se le suma la percepción que tenga el sujeto, noción contemplada en el DSM-V (APA, 20013) y la atribución de significado consecuente.

El brote del COVID-19 comenzó en diciembre del 2019 en China y se declaró pandemia el 11 de marzo del 2020 (OMS, 2020). El contagio es muy rápido, se transmite de persona a persona dentro de los dos metros de proximidad, a través de gotas respiratorias producidas cuando una persona infectada estornuda o tose. Según la OMS (2020) en este momento no habría ni vacuna ni tratamiento específico para esta enfermedad y los sistemas médicos están colapsados; mientras diversos equipos de investigadores de distintos países trabajan sobre la posibilidad de lograr la prevención y tratamiento (Pareja y Luque, 2020).

En China se comenzó a estudiar el impacto psicológico que la pandemia podría estar generando en la población, sobre todo en los profesionales de la salud. Huang et al. (2020), estudiaron el impacto psicológico en el personal de primera línea entre el 7 y el 14 de febrero de 2020, para lo cual aplicaron la Escala de Autoevaluación de Ansiedad (SAS) y la escala de Trastorno por Estrés Postraumático a una muestra de 230 profesionales. Se evidenció que la incidencia de la ansiedad en el personal médico fue del 23,1\% y que fue mayor en el personal femenino. En cuanto al Trastorno por Estrés Postraumático fue del 27,4\%, la cual también fue mayor en el personal femenino.

Lai et al. (2020) realizaron un estudio transversal en 1257 profesionales de la salud de 34 hospitales de China, entre el 29/1/20 y el 3/2/20, con la finalidad de estudiar los niveles de depresión, ansiedad, insomnio y reacción al estrés. El personal informó 
la presencia de síntomas de depresión (50,4\%), de ansiedad (44,6\%), insomnio $(34,0 \%)$ y reacción al estrés $(71,5 \%)$. En este momento hay en el mundo al día de la fecha (17/12/20) 74.728.558 casos confirmados y 1.657 .834 fallecidos; en Argentina 1.524.372 casos confirmados y 41.534 fallecidos .

Los gobiernos de los distintos países afectados han tomado medidas tales como la cuarentena obligatoria, distanciamiento social y la prohibición de viajes, generando con este tipo de decisiones un desequilibrio social y económico. A esta situación se le agrega la sobreinformación y noticias falsas que generan aún mayor miedo e incertidumbre y la discriminación con respecto a los "sospechosos" de haber sufrido el contagio y a los profesionales de la salud. Es importante reflexionar sobre los riesgos para la salud psicofísica de nuestra población y en particular para los profesionales de la salud que son quienes están más expuestos al contagio y a la discriminación social.

La posibilidad de evaluar la ansiedad de la población en estos momentos permite evidenciar la percepción de peligro de los argentinos en esta primera etapa de la cuarentena. De esta manera, es posible orientar las políticas de salud mental hacia una mejor educación y niveles estratégicos de acciones que hagan hincapié en la concientización acerca de los riesgos y/o el manejo de la ansiedad junto a otras medidas de prevención primaria, necesarias para afrontar lo mejor posible esta crisis socio sanitaria mundial.

\section{¿Por qué la ansiedad?}

La ansiedad es una emoción que, dependiendo de la circunstancia, puede ser adaptativa o completamente nociva para el individuo. En el primer caso, permite estar alerta en relación funcional al contexto generando un afrontamiento de la situación y dando lugar a una mejor adaptación. Sin embargo, esta emoción que vela por la supervivencia del individuo puede volverse perjudicial para él: cuando la ansiedad es excesiva en relación con el estímulo que la provoca o cuando tal estímulo no existe, la ansiedad es desadaptativa y se convierte en fuente de malestar.

La ansiedad como una emoción adaptativa, implica la capacidad de informar anticipadamente acerca de las características de los eventos por venir. Esto permite la mejor organización de todo el aparato cognitivo, emocional y pragmático del individuo, ante dichas situaciones de incertidumbre, con la debida selección de estrategias de afrontamiento. Implica una serie de reacciones fisiológicas, experiencias y expresiones emocionales y orientaciones comportamentales que presenta el hombre ante una situación de conflicto (Márquez, 2004). Se torna patológica cuando su intensidad, duración y frecuencia de aparición es exagerada en relación al estímulo y, por lo tanto, se generan conductas inapropiadas (Cía, 2007).

La Asociación Psiquiátrica Americana (2013) define a la ansiedad como aprensión, tensión o dificultad que surge por la anticipación de un peligro cuya fuente es desconocida. En tanto, Lazarus (2000) se refiere a la ansiedad como una relación 
particular entre el individuo y el entorno que es evaluado por éste como amenazante o desbordante de sus recursos y que pone en peligro su bienestar. Navas (1989), en un intento de precisar la ansiedad, la diferencia de otros estados. La explica como un estado emocional, un compuesto o mezcla de sentimientos, conductas y reacciones o sensaciones fisiológicas. Expone que, en el aspecto subjetivo, la ansiedad es un sentimiento o emoción única que es cualitativamente diferente de otros estados emocionales tales como tristeza, depresión, coraje o pesadumbre y agrega que se caracteriza por varios grados de sentimientos de aprensión, temor, terror o nerviosismo. Papalia (1994) refiere a la ansiedad como un estado caracterizado por sentimientos de aprensión, incertidumbre y tensión, más relacionados con la anticipación de una amenaza, real o imaginaria. Postula que los individuos reaccionan con grados de ansiedad variables, que dependen de su propia predisposición a padecer ansiedad y del tipo de amenaza a la que responden.

Wolpe (1981) define a la ansiedad como una respuesta autónoma de un organismo individual concreto después de la presentación de un estímulo nocivo; en términos de aprendizaje, la ansiedad sería tanto una respuesta condicionada como una respuesta incondicionada, pudiendo ser las respuestas de ansiedad ante los estímulos condicionados superiores incluso a las producidas antelos estímulos incondicionados. Lewis (1980) habla de la ansiedad como una emoción desagradable vinculada al miedo y orientada hacia el futuro relacionado a una amenaza, la cual puede ser real o no, pero si lo es, la respuesta es desproporcionada, e irracional. También se la define como una reacción emocional de aprensión, tensión, preocupación, activación y descarga del sistema nervioso autónomo (Spielberger et al., 1984). La ansiedad es un proceso psicobiológico complejo, dentro del cual se distingue a la ansiedad como un estado transitorio por un lado y por el otro, como un rasgo que permanece estable en determinadas personas (Spielberger, 1972).

La ansiedad estado es un sentimiento subjetivo de tensión y nerviosismo junto a una activación del sistema nervioso autónomo, frente a una situación amenazante. Vallejo Ruiloba (1998) sintetiza esto como la valoración del estado mental en tiempo presente del cual habla Spielberger. Por otro lado, la ansiedad rasgo hace referencia a las características de personalidad que hacen a algunas personas a ser más propensas que otras a padecer síntomas de ansiedad (Vallejo Ruiloba, 1998). Spielberger, Pollance y Worden (1984) mencionan que las personas con rasgos de ansiedad son más vulnerables al estrés, percibiendo a muchas más situaciones como peligrosas.

La ansiedad comprende tres sistemas de respuesta: cognitivo, fisiológico y motor (Sandín y Chorot, 1995). Dentro de lo cognitivo estarían las percepciones subjetivas vinculadas a los estímulos y estados asociados con la ansiedad (miedo, preocupaciones, pensamientos intrusivos etc.). Con respecto a lo fisiológico se da un incremento de la actividad del sistema nervioso autónomo. Según el DSM-IV (APA, 1994), esto se puede manifestar a través de diarrea, palpitaciones, hipertensión, temblores, malestar gástrico etc. En lo que tiene que ver con lo conductual se daría a 
través de gestos, agitación y respuestas de escape y evitación.

Clark y Beck (2012) conceptualizaron a la ansiedad como un sistema complejo de respuesta conductual, fisiológica, afectiva y cognitiva que se dispara al anticipar sucesos percibidos como imprevisibles, incontrolables y potencialmente amenazantes para los intereses vitales de una persona. En la presente investigación se partió de la hipótesis de que la ansiedad en este contexto tan complejo y de tanta incertidumbre se dispararía a niveles muy elevados.

\section{Objetivos y metodología}

Con respecto a los objetivos generales, se planteó evaluar la ansiedad con respecto al COVID-19 en el contexto de Pandemia en Argentina. En los específicos se esperó medir la ansiedad en: los grupos de factores de riesgo, las personas que estarían expuestas al contagio por su trabajo, los profesionales de la salud y la población en general.

La investigación pudo categorizarse como un estudio no experimental transversal (Hernandez Sampieri, Baptista, Fernandez Collado, 2010). El muestreo fue de carácter no probabilístico, de voluntarios. El instrumento de recolección utilizado fue un cuestionario autoadministrado divulgado por redes sociales durante 6 días a partir del 26/3/20, esto es, luego de la primera semana de declarada la cuarentena en el territorio de la República Argentina. La muestra estuvo conformada por 2055 personas, de las cuales 448 fueron excluidas del estudio ya que las escalas utilizadas no eran aplicables a su contexto por ser extranjeros.

Al tratarse de un muestreo no probabilístico, no fue posible hacer inferencia a la población total de Argentina, por lo que el análisis fue únicamente de tipo descriptivo y las conclusiones aplican a la muestra relevada. Si bien la cantidad de respuestas obtenidas fue importante, la conformación según género no refleja la composición demográfica del país (el 75\% de respondentes fueron mujeres) y por lo tanto los resultados podrían estar sesgados.

El cuestionario estuvo constituido por preguntas relativas a: (i) si el participante pertenecía al grupo de riesgo para el Coronavirus y si por su trabajo estaría expuesto al contagio; (ii) si el participante era profesional de la salud y de ser así, en qué sitio trabajaba; (iii) el Cuestionario de Ansiedad Estado-Rasgo (STAI), que es un instrumento psicométrico que comprende 40 ítems distribuidos en dos escalas separadas de autoevaluación (Spielberger, Gorsuch y Lushore, 1970). Estas escalas miden dos conceptos de ansiedad independientes: la ansiedad como estado (condición emocional transitoria) y la ansiedad como rasgo (propensión ansiosa relativamente estable). Cada subescala se conforma por un total de 20 ítems utilizando una escala Likert de 4 puntos según la intensidad (0: nada, 1: un poco, 2: bastante, 3: mucho). La puntuación total en cada una de las subescalas oscila entre 0 y 60 puntos. Esta escala ha sido validada en Argentina (Figueroa, 1991); (iv) variables socio demográficas: género, edad, ocupación, profesión, ciudad, país. 
Para valorar los resultados de los niveles de ansiedad obtenidos en la muestra se compararon los valores medios de los puntajes obtenidos en cada una de las escalas con los baremos de la escala validada en el país, teniendo en cuenta los grupos de edad y sexo de los respondentes.

También se segmentó la muestra según algunas de las variables que caracterizaban a los encuestados para comparar los niveles de Ansiedad Estado y Ansiedad Rasgo entre algunos grupos de interés (grupo de riesgo, profesionales de la salud, modalidad de trabajo).

En todas las tablas, además del promedio aritmético de los valores obtenidos en cada escala, se presentó el desvío estándar de cada variable a los fines de conocer el grado de dispersión de la muestra y del estadístico utilizado para medir los niveles de ansiedad.

Los grupos de riesgo a contraer COVID-19 están determinados en la página oficial del gobierno argentino (www.argentina.gob.ar) y son en su mayoría coincidentes los descritos en la mayoría de páginas y sitios de internet de Argentina y del mundo, a saber:

- El asma, de moderado a grave.

- La enfermedad renal crónica, bajo tratamiento con diálisis.

- Las enfermedades hepáticas crónicas, incluida la cirrosis.

- Las enfermedades pulmonares crónicas, como el enfisema pulmonar obstructivo crónico (EPOC), incluida la fibrosis quística, bronquitis crónica y la fibrosis pulmonar idiopática.

- La diabetes (de tipo 1, 2 o gestacional).

- La inmuno-depresión por diferentes patologías o afecciones, por ejemplo, el tratamiento para el cáncer, déficit del sistema inmunitario, trasplantes, pacientes con VIH con un bajo recuento de células CD4 o sin tratamiento para el VIH, uso prolongado de corticoides y otros medicamentos que debilitan el sistema inmunitario.

- Los adultos mayores (65 años o más).

- Los trastornos de la hemoglobina como anemia falciforme y la talasemia.

- Adultos mayores que viven en asilos de ancianos o establecimientos de cuidados a largo plazo.

- Las patologías cardíacas, incluidas las enfermedades cardiacas congénitas, las cardio-miopatías, la insuficiencia cardiaca, la enfermedad de las arterias coronarias y la hipertensión pulmonar. 


\section{- La obesidad grave (IMC de 40 o más).}

Por último y a los fines de evaluar la consistencia interna del cuestionario, se calculó el índice de Cronbach para cada una de las escalas.

\section{Resultados}

En la muestra correspondiente a los argentinos, $75 \%$ resultaron mujeres y $25 \%$ hombres, siendo la conformación de grupos de edad similar en ambos géneros. Las edades iban desde los 15 a los 85 años con una media de 44,26 años y una mediana de 43 .

En función de los grupos de interés del trabajo, la muestra estuvo conformada de la siguiente manera: el 33,4\% de los participantes declaró pertenecer a algún grupo de riesgo, mientras que un $8,5 \%$ dijo no estar seguro. El $83,9 \%$ de todos los que respondieron a la encuesta no estaba desarrollando en dicho momento un trabajo en el que tuviera riesgo de contagio. El 19,3\% era profesional de la salud.

Tanto para los hombres como para las mujeres y para todos los grupos etarios, los puntajes de ambas escalas (AE y AR) se corresponden con percentiles que están por debajo del primer cuartil de la distribución de los baremos. Esto significa que todos los grupos presentan un nivel bajo de ansiedad. No obstante, dentro de esos bajos niveles de ansiedad se pudo observar algunas diferencias cuando se comparan los límites superiores del intervalo de confianza para el nivel medio de cada escala, teniendo en cuenta su variabilidad, con los perceptibles tabulados. Así podemos observar que las mujeres presentan un nivel de ansiedad mayor al de los hombres.

Se compararon los grupos de interés, sabiendo que en todos los casos los niveles se corresponden con valores de ansiedad bajo en ambas escalas (percentiles menores a 20 en la escala Estado y menores a 7 en la escala Rasgo para todos los grupos). No se advirtieron diferencias entre quienes se consideran o no grupos de riesgo (tanto en hombres como en mujeres). En cambio, en aquellos que afirmaron no saber si pertenecían o no a un grupo de riesgo los valores de AE fueron superiores.

Las personas que sintieron que en su trabajo se encontraban en riesgo de contagio, aumentaron el AE, tanto en los hombres como en las mujeres (en el caso de éstas el aumento fue mayor). Los varones evidenciaron un valor más alto cuando no sabían fehacientemente si estaban en riesgo de contagio o no. En la escala de AR no se observaron diferencias en el grupo de las mujeres entre quienes estaban o no en riesgo de contagio. Sí, hubo una variación entre los hombres, disminuyendo el nivel de AR en el grupo de los que sabían si estaban en riesgo (comportamiento inverso a los que ocurre con la $\mathrm{AE}$ ).

Se distinguieron otros dos grupos: profesionales y no profesionales de la salud. Los profesionales de la salud presentaron niveles de AE superiores a los de los noprofesionales de la salud. En la escala AR el comportamiento fue inverso.

En el grupo de los hombres, los que presentaron AE más alta fueron los profesionales 
de salud mental seguidos por los odontólogos y en último lugar los médicos; con respecto a la $\mathrm{AR}$ se dio más elevada en los profesionales de salud mental.

Tabla 1. Niveles de AE y AR, según grupo etario (entre paréntesis figuran los percentiles con que se corresponden)

\begin{tabular}{rlrrrr}
\hline & & \multicolumn{2}{c}{ Hombres } & \multicolumn{2}{c}{ Mujeres } \\
\cline { 3 - 6 } & & \multicolumn{1}{c}{ AE } & \multicolumn{1}{c}{ AR } & \multicolumn{1}{c}{ AE } & \multicolumn{1}{c}{ AR } \\
\hline 19 a 39 & N & 195 & 193 & 593 & 589 \\
& Media & 22,4667 & 19,2539 & 25,7757 & 21,0306 \\
& & $(8)$ & $(3)$ & $(17)$ & $(3)$ \\
& Desv. Típ. & 9,29376 & 9,32977 & 10,03298 & 9,71336 \\
40 a 49 & N & 132 & 131 & 431 & 429 \\
& Media & $22,6742(12)$ & $17,1603(1)$ & $26,0789(19)$ & $19,9347(0)$ \\
& Desv. Típ. & 8,99915 & 8,39763 & 10,19203 & 9,40051 \\
50 a 69 & N & 149 & 148 & 458 & 458 \\
& Media & $20,5436(9)$ & $16,4459(3)$ & $21,8908(12)$ & $16,9192(<7)$ \\
& Desv. Típ. & 9,70197 & 9,18612 & 9,74989 & 9,65737
\end{tabular}

Tabla 2. Niveles de AE y AR según pertenencia a Grupo de Riesgo o Riesgo de Contagio

\begin{tabular}{|c|c|c|c|c|c|c|}
\hline & & & Hon & & $\mathrm{Mu}$ & \\
\hline & & & $\mathrm{AE}$ & AR & $\mathrm{AE}$ & AR \\
\hline${ }_{¿}$ Es grupo de & Sí & $\mathrm{N}$ & 173 & 170 & 513 & 509 \\
\hline riesgo? & & Media & 21,0983 & 16,1647 & 24,5556 & 18,6817 \\
\hline & & Desv. Típ. & 9,84689 & 9,27916 & 10,64196 & 9,93962 \\
\hline & No & $\mathrm{N}$ & 289 & 288 & 905 & 902 \\
\hline & & Media & 21,3426 & 18,1736 & 24,1812 & 19,6774 \\
\hline & & Desv. Típ. & 8,87472 & 9,16122 & 9,96700 & 9,88273 \\
\hline & No sé & $\mathrm{N}$ & 47 & 46 & 127 & 127 \\
\hline & & Media & 27,5319 & 20,3696 & 27,9843 & 21,5669 \\
\hline & & Desv. Típ. & 8,47721 & 6,61768 & 9,56928 & 9,36185 \\
\hline ¿Tiene riesgo & Sí & $\mathrm{N}$ & 90 & 90 & 207 & 206 \\
\hline de contagio? & & Media & 22,6667 & 16,1222 & 28,2609 & 19,3058 \\
\hline & & Desv. Típ. & 9,76234 & 8,67105 & 10,91892 & 9,00020 \\
\hline & No & $\mathrm{N}$ & 409 & 404 & 1314 & 1308 \\
\hline & & Media & 21,5355 & 18,0000 & 24,0190 & 19,4709 \\
\hline & & Desv. Típ. & 9,25480 & 9,17625 & 9,99972 & 10,00382 \\
\hline & No sé & $\mathrm{N}$ & 10 & 10 & 24 & 24 \\
\hline & & Media & 26,4000 & 19,6000 & 26,0000 & 23,0000 \\
\hline & & Desv. Típ. & 8,30261 & 7,30601 & 8,90530 & 10,28718 \\
\hline
\end{tabular}


Tabla 3. Comparación de nivel medio de AE y AR entre profesionales de la salud y otros

\begin{tabular}{llcr}
\hline & & AE & \multicolumn{1}{c}{ AR } \\
\hline Es profesional de la salud & Media & 25,276 & 18,2611 \\
& Desv. Típ. & 10,47548 & 8,61417 \\
\multirow{2}{*}{ No es profesional de la salud } & Media & 23,6952 & 18,2611 \\
& Desv. Típ. & 9,91366 & 8,61417
\end{tabular}

En el grupo de las mujeres los niveles de AE resultaron más altos en las acompañantes terapéuticas, enfermeras y profesionales de laboratorio, le siguieron las médicas y odontólogas y por último las profesionales de salud mental. Los niveles de AR más elevados se vieron en las acompañantes terapéuticas y los más bajos en las enfermeras, kinesiólogas y profesionales de salud mental. Es decir que, en el caso de las mujeres, las enfermeras a pesar de presentar AE más elevada, evidenciaron escasos niveles de AR (baja vulnerabilidad).

Con respecto a los profesionales que en este momento trabajan en forma online y los que trabajan en forma presencial, se pudo observar que en los hombres hay un mayor nivel de AE y de AR para los que trabajan en forma online. Mientras que, en las mujeres, la AE y la AR se evidenció más elevada en aquellas que están trabajando en forma presencial.

Durante el relevamiento, el presidente de la Nación anunció la extensión de la cuarentena. Se realizó una comparación de las medias de ambas escalas y resultó que ambos promedios resultaron inferiores para el periodo posterior al anuncio, tanto para los hombres como para las mujeres.

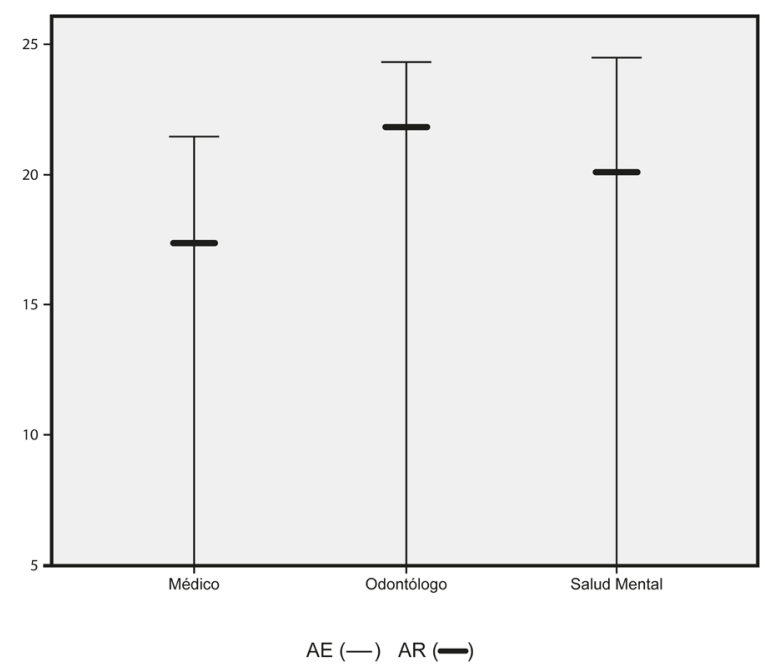

Figura 1. Niveles medios de AE y AR para los hombres, según su especialidad 


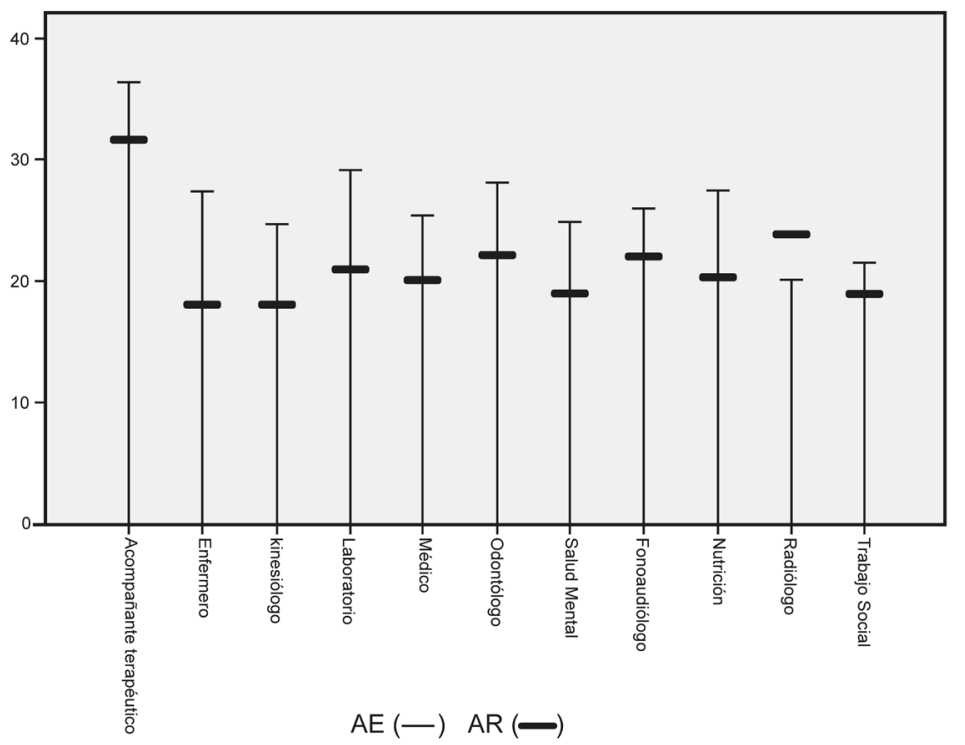

Figura 2: Niveles medios de AE y AR para las mujeres, según su especialidad

En cuanto a las propiedades psicométricas, el cuestionario posee una buena consistencia interna para ambas escalas: el alfa de Cronbach para la escala AE va desde 0,879 y 0,895 , mientras que para la escala de AR toma valores entre 0,863 y 0,864 .

Tabla 4. Niveles de AE y AR para los hombres y mujeres según modalidad de trabajo

\begin{tabular}{rlrrrr}
\hline \multicolumn{2}{c}{ Modalidad } & \multicolumn{2}{c}{ Hombres } & \multicolumn{2}{c}{ Mujeres } \\
\cline { 3 - 7 } \multicolumn{2}{r}{ Presencial } & AE & AR & \multicolumn{1}{c}{ AE } & \multicolumn{1}{c}{ AR } \\
& N & 28 & 28 & 109 & 108 \\
& Media & 22,4286 & 17,9286 & 27,9358 & 18,9722 \\
\multirow{3}{*}{ Online } & Desv. Típ. & 9,69700 & 8,35299 & 10,86642 & 7,92306 \\
& N & 19 & 19 & 144 & 143 \\
& Media & 22,9474 & 18,3158 & 23,8681 & 16,7902 \\
& Desv. Típ. & 9,29441 & 7,96557 & 9,39963 & 7,81643
\end{tabular}

\section{Discusión}

La pandemia del COVID-19 y la cuarentena constituyen dos situaciones generadoras de crisis con componentes de altos niveles de incertidumbre, ansiedad y estrés (Cedeño et al., 2020; Ozamiz-Etxebarria et al., 2020: Moreno-Proaño, 2020). La crisis puede ser definida como un estado de máxima tensión en un sistema, un desajuste crítico que altera su estabilidad. Es un desvío del curso regular de las interacciones de un sistema (Ceberio y Watzlawick, 1998, De Alba y Puiggrós, 1991) y existen crisis esperadas e inesperadas. Las primeras son, de acuerdo con el contexto, las 
que forman parte de la evolución de los sistemas, mientras que las inesperadas muestran situaciones que exceden el marco de lo habitual. Este último es el caso de la pandemia del COVID-19. El estrés, como variable ineludible en este tipo de situaciones (Peiró y Salvador, 1993; Lazarus, 2000), activa el eje hipófiso-adrenal para que se produzca una cuota adicional de cortisol y poder sobrellevar el momento crítico (Gaudlitz, Plag, Dimeo y Ströhle, 2015; Llaneza Alvarez, 2009; Pérez Martín, 2001). La ansiedad es la emoción resultante de la ecuación crisis-estrés y evaluarla fue la finalidad de este estudio.

A los 10 días de iniciada la cuarentena en la Argentina (a partir del 20 de marzo de 2020) se inició una exploración acerca de la ansiedad que generaba la pandemia y el confinamiento obligatorio. Se diferenció la ansiedad de rasgo (AR) y la ansiedad de estado (AE): cuál era la ansiedad esperable en las personas en tiempos normales y cuál en tiempos de crisis. Por ansiedad de estado (AE) se evalúa un estado emocional transitorio, caracterizado por sentimientos subjetivos que se perciben de manera consciente, de atención y aprensión y por hiperactividad del sistema nervioso autónomo (Spielberg et al., 1999). La ansiedad como rasgo (AR) marca la propensión ansiosa que aparece en las personas con cierta regularidad y se caracteriza por la tendencia a percibir las situaciones de la vida como amenazantes y con ello aumentando el nivel de vulnerabilidad (Spielberg y Díaz Guerrero, 1975; Spielberg et al., 1999).

Lo que destaca la investigación, sobre la base de la ansiedad de rasgo, es la diferencia cualitativa y cuantitativa con la ansiedad de estado en esta situación de la pandemia y el confinamiento consecuente. Si bien se obtuvieron diferencias entre ambos niveles de ansiedad, la AE fue baja (en ningún caso supera el percentil 20), lo que guarda relación con la baja AR (siempre por debajo del percentil 7) que se evidenció en la muestra en general. Frente a estos datos se observó que la AR de los argentinos fué más baja de lo esperado al igual que la $\mathrm{AE}$ ante esta crisis. Uno de los factores que puede tomarse en cuenta como hipótesis dado el bajo nivel ansiógeno puede remitirse a la inestabilidad del contexto social, político y económico argentino que genera incertidumbre e incrementa el nivel de adaptabilidad para la supervivencia. Algunas investigaciones revelan diferentes sistemas inestables en el poder político, en las políticas económicas y sociales, la reiteración de fórmulas ineficaces para solución de la inestabilidad y cómo a pesar de la capacitación, los jóvenes tienen dificultades de encontrar su lugar laboral (Carrizo y Galván, 2017; Nemiña y Val, 2018; Malamud y De Luca, 2016; Castellani y Heredia, 2020; Rubio y Fachal, 2018). Podría entenderse entonces el contexto de la pandemia y cuarentena como un factor más de la inestabilidad sistematizada en la Argentina y con ello una menor irrupción ansiógena en el argentino/a.

Si bien los niveles de ansiedad en general fueron bajos, se pudieron observar algunas diferencias. Por un lado, las mujeres presentaron mayor ansiedad que los hombres (ver Tabla 1). Luego, se evidencio mayor ansiedad en los profesionales de la salud (ver Tabla 3) tal como se pudo ver en estudios realizados en China (Lai et al, 2020)- , en los trabajadores expuestos al contagio (ver Tabla 2) y en los grupos de riesgo (ver Tabla 
2). Tanto en los profesionales como en los trabajadores expuestos, el hecho de que la ansiedad sea más elevada guarda cierta lógica emocional, dado el riesgo al contagio por el nivel de exposición y la labilidad por las deficiencias orgánicas que facilitan la infección. Con respecto al grupo de las mujeres, que presentaron un alza de la ansiedad, cabe preguntarse si éstas han multiplicado la responsabilidad de las tareas del hogar y generando de esta forma este resultado. A pesar de que en los tiempos actuales ha cambiado el rol de la mujer (por ejemplo, la reivindicación de la mujer en tareas que sólo ocupaban los hombres, o la ruptura de la función absoluta de "ama de casa"), las actividades domésticas lamentablemente continúan siendo patrimonio femenino. Hay "colaboración" del hombre, pero no un desarrollo equitativo y compartido de las tareas (Ceberio, 2013a, 2013 b; Reyes, 2019; Zavaleta, 2019).

Dentro de los profesionales de la salud, en el grupo de los hombres se observó una mayor vulnerabilidad y ansiedad en los profesionales de la salud mental (ver Figura 1) (a pesar de que la atención presencial no estaba habilitada, o sea, que las consultas eran siempre online). En cambio, en las mujeres la AE fue más elevada en las enfermeras, las profesionales de laboratorio y acompañantes terapéuticas, a las que le siguieron las médicas y odontólogas y, por último, las profesionales de salud mental (ver Figura 2). Todos niveles de actuación profesional de contacto, de trabajo cuerpo a cuerpo, lo que determina un mayor riesgo y, por lo tanto, mayor ansiedad. Los niveles de AR más elevados, por ende, mayor propensión ansiógena, se vieron en las acompañantes terapéuticas y los más bajos, en las enfermeras, kinesiólogas y profesionales de salud mental.

En este grupo, notablemente los hombres presentaron mayor ansiedad al trabajar en forma online y las mujeres mayor ansiedad al trabajar en forma presencial (ver Tabla 4). Es significativo que los hombres presenten mayor ansiedad en el trabajo online, puesto que la pantalla anula, por así decirlo, el riesgo al contagio (como factor de ansiedad), aunque no puede determinarse unívocamente a la presencialidad como único factor ansiogénico. Otro dato que completa esta evaluación es la "consciencia de riesgo": cuando la persona siente que en su trabajo se encuentra en riesgo de contagio, aumenta el AE, tanto en los hombres como en las mujeres -y en el caso de ellas, el aumento es mayor-. Los varones evidenciaron un valor más alto cuando no sabían fehacientemente si estaban en riesgo de contagio o no (mayor incertidumbre).

Después del anuncio del presidente sobre la extensión de la cuarentena (29/03/20), se pudo observar en la población un descenso de los niveles de ansiedad, cuando podrían haberse exacerbado por el fastidio, la inseguridad y la incertidumbre que implica el encierro (Gallegos et al., 2020; Ceberio, 2020). Cabe la posibilidad que el descenso de los niveles de AR y AE, entre las múltiples hipótesis que pueden barajarse y más allá de niveles de adaptabilidad contextual naturales, pueda deberse a la efectividad de la puesta en práctica de una serie de "facilitadores". Estos son consejos y sugerencias psicoeducativas que se desarrollaron desde los inicios de la cuarentena, principalmente de psicólogos, y que operaron como factores amortiguadores de la angustia y ansiedad que detonaban la presencia del virus y el consecuente miedo y encerramiento (Ceberio, 2020). 
Otras investigaciones que también exploraron emociones en el confinamiento del COVID-19, principalmente en ansiedad, encontraron resultados diversos. OzamizEtxebarria, et al. (2020) midieron variables de ansiedad, estrés y depresión y los resultados mostraron niveles de sintomatología bajos en general, pero en población joven y con enfermedades crónicas ha referido sintomatología más alta que el resto y han observado un mayor nivel de sintomatología a partir del confinamiento. Arias Molina, et al. (2020) estudiaron las variables ansiedad -también aplicando el STAI-, depresión, estrés e ideas suicidas. En los resultados se vieron alteraciones por ansiedad y depresión como estado, altos niveles de estrés y $0 \%$ en ideación suicida. Refieren los autores que la ansiedad supera la capacidad adaptativa y en muchos casos conduce a la práctica de actividades no rutinarias como la ingesta de bebidas alcohólicas para tolerar la situación. Huarcaya-Victoria (2020) informó la presencia de ansiedad, depresión y estrés en la población general y detectó problemas de salud mental, especialmente en las profesionales mujeres -enfermeras y personal sanitario que trabaja con contagiados del virus-. Cedeño, et al (2020) desarrollaron una revisión sistemática en el arco de 8 años y centrándose más en este último, de la que resultaron 34 artículos que destacaron el estrés, miedo, ansiedad y depresión como emociones principales en la pandemia del COVID-19. Moreno-Proaño (2020) afirmó que durante la pandemia del COVID-19 se ha producido una mayor demanda de atención psicológica a raíz de síntomas de ansiedad como insomnio, inapetencia, preocupación, cansancio. Los autores destacaron la aparición de pensamientos distorsionados en la ansiedad que obstaculizan las acciones cotidianas. Otros autores mencionaron la presencia de ansiedad y miedo ante la muerte en contexto de COVID-19 (Tomás-Sabato, 2020), ansiedad y depresión (Galindo-Vazquez et al., 2020), estrés, ansiedad, síntomas depresivos, insomnio, negación e ira (LozanoVargas, 2020) y manifestaciones ansiógenas en diferentes situaciones y poblaciones (Soca Guzmán, 2020; Saravia-Bartra, et al. 2020; Santamaría, et al 2020; Jurado, et al, 2020; Jerves Mora, 2020; Sanchez y Aguila, 2020).

\section{Conclusión}

Los niveles de ansiedad en la muestra de la población argentina fueron más bajos de lo esperado, tanto en lo que es ansiedad estado como en la ansiedad rasgo. Si bien la ansiedad estuvo presente, los niveles bajos en la diferenciación en relación a la AR podrían referirse a numerosas razones: en primer lugar a los niveles de adaptación del argentino medio por la sistematización de su contexto inestable.

En segundo lugar, podría suponerse que la efectividad de los consejos y la psicoeducación para tolerar el confinamiento que salieron a brindar los profesionales de la salud mental a través de las redes sociales y los medios de comunicación pudieron haber operado como un poderoso distractor para sobrellevar esos primeros días. En paralelo, continúan desarrollándose situaciones productoras de ansiedad: la incertidumbre a múltiples niveles, el futuro incierto, el miedo al contagio, los factores económicos y la inestabilidad laboral, entre otros. 
Se espera que la ansiedad se eleve con el correr de los días de confinamiento, por diferentes razones tales como el encierro mismo junto con el desconocimiento sobre su fecha de fin, el aumento de la sobreinformación, las noticias falsas y las diversas teorías sobre el virus, el incremento del miedo, las diversas alternativas de convivencia, el trabajo en el hogar, la escolaridad virtual y sus complicaciones, el agotamiento de recursos facilitadores, entre otros factores.

Por lo mencionado, el equipo de investigación tiene planificado una segunda administración a partir del día noventa de cuarentena, teniendo en cuenta que la pandemia recién comienza en nuestro país y que es una gran limitación la rapidez con la que se están produciendo los cambios de esta situación en el mundo.

\section{Referencias}

American Psychiatric Association. (2013). Diagnostic and statistical manual of mental disorders (DSM5). American Psychiatric Pub.

American Psychiatric Association. (1994) Diagnostic and statistical manual of mental disorders (DSM-4). American Psychiatric Pub.

Arias Molina, Y., Herrero Solano, Y., Cabrera Hernández, Y., Guyat, D. C. y Mederos, Y. G. (2020). Manifestaciones psicológicas frente a la situación epidemiológica causada por la COVID-19. Revista Habanera de Ciencias Médicas, 19 (Supl.1)

Bertalanffy, L. (1968) Teoría general de los sistemas. Fondo de Cultura Económica.

Carrizo, C., y Galván, C. (2017). Presidencialismo y conflictos políticos en Argentina: sobre la inestabilidad política a nivel nacional $\mathrm{y}$ provincial (1983-2006). Colección, 17, 35-113.

Castellani, A. G., y Heredia, M. (2020). La reproducción fallida de las elites: Inestabilidad y transformaciones de las elites empresariales argentinas entre 1976 y 2015. RES. Revista Española de Sociología, 29(3), 467-486. нттр:// DOI.ORG/10.22325/FES/RES.2020.30

Ceberio, M.R. (2011) Nuevas estructuras de familia, nuevos desafíos. En M. R. Ceberio y H. Serebrinsky (Eds.), Dentro y fuera de la caja negra (pp. 90-110). Psicolibro.

Ceberio, M. R. (2013) Nuevas estructuras de familiares, nuevos desafíos terapéuticos. Viejas y nuevas familias. En M. R. Ceberio y H. Serebrinsky (Eds.), Dentro y fuera de la caja negra.
Ceberio, M. (2013). El cielo puede esperar. Ediciones Morata.

Ceberio, M. R. (2014) Los juegos del miedo. Hacia un modelo integrador en el tratamiento de los trastornos de pánico. Pensamiento Sistémico, 341-374.

Ceberio, M. R. (2020). Psicólogos en el frente: la atención durante la crisis del Covid-19. De las emociones tóxicas a la salud psicológica. Archivos de Medicina (Manizales), 21(1). HTTPS:// DOI.ORG/10.30554/ARCHMED.21.1.3941.2021

Ceberio, M. R. y Watzlawick, P. (1998). La construcción del universo. Herder.

Cedeño, N. J. V., Cuenca, M. F. V., Mojica, Á. A. D. y Portillo, M. T. (2020). Afrontamiento del COVID-19: estrés, miedo, ansiedad y depresión. Enfermería Investiga, 5(3), 63-70. HTTP://DX.DOI. ORG/10.31164/ENF.INV.V5I3.913.2020

Cía, A. H. (2007). La ansiedad y sus trastornos: Manual diagnóstico y terapéutico. En A. Cía (Ed.), La ansiedad y sus trastornos: manual diagnóstico y terapéutico (pp. 494-494). Polemos.

Clark, D. A. y Beck, A. T. (2012). Terapia cognitiva para trastornos de ansiedad (Trad., J. Aldekoa). Desclee de Brouwer.

De Alba, A. y Puiggrós, A. (1991). Curriculum: crisis, mito y perspectivas. Miño y Davila Editores.

Figueroa, N. (1991). Ansiedad. Algunas concepciones teóricas y su evaluación. En M. Casullo, N. Figueroa y Aszkenazi, Teoría y técnicas de evaluación psicológica (pp. 123-135). Psicoteca. 
Galindo-Vázquez, O., Ramírez-Orozco, M., Costas-Muñiz, R., Mendoza-Contreras, L. A., Calderillo-Ruíz, G. y Meneses-García, A. (2020). Síntomas de ansiedad, depresión y conductas de autocuidado durante la pandemia de COVID-19 en la población general. Gaceta Médica, 156, 298 305.

Gallegos, M., Zalaquett, C., Luna Sanchez, S. E., Mazo-Zea, R., Ortiz-Torres, B., Penagos-Corzo, J., Portillo, N., Torres Fernandez, I., Urzúa, A., Consoli, M., Polanco, F. A. ,Florez, A., y López Miranda, R.(2020). Cómo afrontar la pandemia del Coronavirus (Covid-19) en las Américas: recomendaciones y líneas de acción sobre salud mental. Interamerican Journal of Psychology, 54(1), 1-28. HTTPS://DOI.ORG/10.30849/RIPIJP. V54I1.1304

Gaudlitz, K., Plag, J., Dimeo, F. y Ströhle, A. (2015). Aerobic exercise training facilitates the effectiveness of cognitive behavioral therapy in panic disorder. Depression and Anxiety, 32(3), 221-228. $\quad$ HTTPS://DOI.ORG/10.30849/RIPIJP. $\mathrm{V} 54 \mathrm{I1} .1304$

Hernandez-Sampieri, R., Fernández, C. y Batista, P. (2010). Metodología de la investigación (5ta. edición). Mc Graw Hill.

Huarcaya-Victoria, J. (2020). Consideraciones sobre la salud mental en la pandemia de COVID-19. Revista Peruana de Medicina Experimental y Salud Pública, 37(2). HTTPS://DOI.ORG/10.17843/ RPMESP.2020.372.5419

Huang, J.Z., Han, M.F., Luo, T.D., Ren, A.K., y Zhou, X.P. (2020). Mental Health survey of 230 medical staff in a tertiary infectious disease hospital for COVID-19. Chinese Journal of Industrial Hygiene and Occupational Diseases, 38, E001-E001. HTTPS://DOI.ORG/10.3760/ CMA.J.CN121094-20200219-00063

Jerves Mora, R. S. (2020). Pandemia y ansiedad social. Revista Facultad de Ciencias Médicas Universidad Cuenca, 13-16. HTTPS://DOI. ORG/10.18537/RFCM.38.01.03

Jurado, M. D. M. M., Herrera-Peco, I., del Carmen Pérez-Fuentes, M. y Linares, J. J. G. (2020). Análisis de la amenaza percibida por la COVID-19 en población española. Atención Primaria, 52(7), 515-516. HTTPs://DOI. ORG/10.1016/J.APRIM.2020.05.001

Lai J., Ma, S., Wang, Y., Cai, Z., Hu, J., Wei, N., y Tan, H. (2020). Factors associated with mental health outcomes among health care workers exposed to coronavirus disease 2019. JAMA Network Open, 3(3), e203976-e203976. HTTPS://DOI.ORG/10.1001/ JAMANETWORKOPEN.2020.3976
Lang, P. J. (1968). Fear reduction and fear behavior: Problems in treating a construct. Research in Psychotherapy Conference, 3, 90-102.

Lazarus, R. S. (2000). Estrés y emoción. Manejo e implicaciones en nuestra salud. Desclée de Brouwer.

Lewis, A. (1980). Problems presented by the ambiguous word anxiety as used in psychopathology. En G. D. Burrows y B. Davies (Eds.), Studies on anxiety (pp. 1-15).

Llaneza Alvarez, J. (2009). Ergonomía y psicosociología aplicada: manual para la formación del especialista (12a edicion). Lex Nova.

Lozano-Vargas, A. (2020). Impacto de la epidemia del Coronavirus (COVID-19) en la salud mental del personal de salud y en la población general de China. Revista de Neuro-Psiquiatría, 83(1), 5156. HTTP://DX.DOI.ORG/10.20453/RNP.V83I1.3687

Malamud, A. y De Luca, M. (2016). ¿ Todo sigue igual que ayer? Continuidad y ruptura en el sistema de partidos argentino (1983-2015).En F. Freidenberg (Ed.), Los sistemas de partidos en América Latina (1978-2015). Tomo 2. Cono Sur y Países Andinos (pp. 27-68).

Márquez, M. (2004). La ansiedad y sus trastornos: más allá de la psicobiología. En A. Lopez Mato, A. (Compiladora). Psiconeuroinmunoendocrinología. Nuevos dilemas para viejos paradigmas. Viejos dilemas para nuevos paradigmas. Polemos.

Moreno-Proaño, G. (2020). Pensamientos distorsionados $\mathrm{y}$ ansiedad generalizada en COVID-19. CienciAmérica, 9(2), 251-255. HTTP://DX.DOI.ORG/10.33210/CA.V9I2.314

Navas, J. (1989). Ansiedad en la toma de exámenes: algunas explicaciones cognoscitivasconductuales. Revista Aprendizaje $y$ Comportamiento, 7(1), 21-41.

Nemiña, P., y Val, M. E. (2018). El conflicto entre la Argentina y los fondos buitres. Consecuencias sobre los procesos de reestructuración de deuda soberana. Cuadernos de Economía Crítica, 5(9), 45-68.

Organización Mundial de la Salud (OMS) (2020). Alocución de apertura del Director General de la OMS en la rueda de prensa sobre la COVID-19 celebrada el 11 de marzo de 2020. Disponible en: HTTPS://BIT.LY/2WKT9AJ 
Ozamiz-Etxebarria, N., Dosil-Santamaria, M., Picaza-Gorrochategui, M., y IdoiagaMondragon, N. (2020). Niveles de estrés, ansiedad y depresión en la primer fase del brote del COVID-19 en una muestra recogida en el norte de España. Cadernos de Saúde Pública, 36, e00054020. HTTPS://DOI.ORG/10.1590/0102$311 \mathrm{X} 00054020$

Papalia, D. (1994). Psicologia. McGraw-Hill.

Pareja Cruz, A., y Luque Espino, J. C. (2020). Alternativas terapéuticas farmacológicas para COVID-19. Horizonte Médico (Lima), 20(2). HTTP://DX.DOI.ORG/10.24265/HORIZMED.2020. V2ON2.13

Pérez Martín, J. (2001). Deficiencia mental y familiar. Promi.

Peiró, J. M. y Salvador, A. (1993). Desencadenantes del estrés laboral (Vol. 2). Eudema.

Reyes, S. Á. R. (2019). Transformaciones generadas por las marcas en el empoderamiento femenino y roles de la mujer. Investigación en Comunicación Publicitaria, 5.

Rubio, M. B., y Fachal, M. N. (2018). Principales tendencias en el vínculo educación y empleo: los jóvenes en la Argentina de la postconvertibilidad (2004-2014). EntreDiversidades, 10, 59-98. HTTPS://DOI.ORG/10.31644/ED.10.2018.A03

Sánchez, M. V., y Águila, H. D. (2020). COVID-19: respuestas psicológicas y maneras de gestionarlas. Revista del Hospital" Dr. Emilio Ferreyra", 1(1), e33-e34. HTTPS://DOI.ORG/10.5281/ ZENODO. 3856407

Sandín, B. y Chorot, P. (1995). Concepto y categorización de los trastornos de ansiedad. Manual de Psicopatología, 2, 53-80.

Santamaría, M. D., Ozamiz-Etxebarria, N., Rodríguez, I. R., Alboniga-Mayor, J. J., y Gorrotxategi, M. P. (2020). Impacto psicológico de la COVID-19 en una muestra de profesionales sanitarios españoles. Revista de Psiquiatría $y$ Salud Mental. HTTPS://DOI.ORG/10.1016/J. RPSM.2020.05.004

Saravia-Bartra, M. M., Cazorla-Saravia, P., y Cedillo-Ramirez, L. (2020). Nivel de ansiedad de estudiantes de medicina de primer año de una universidad privada del Perú en tiempos de Covid-19. Revista de la Facultad de Medicina Humana, 20(4), 568-573. HTTPs://DOI. ORG/10.25176/RFMH.v20I4.3198
Soca Guzmán, C. A. (2020). Frecuencia de miedo, ansiedad y depresión en gestantes en el contexto COVID-19 en un hospital general de LimaMetropolitana. Repositorio de la Universidad Cayetano Heredia. HTTP://REPOSITORIO.UPCH. EDU.PE/HANDLE/UPCH/8468

Spielberger, C. (1972). Anxiety as an emotional state. Anxiety. Current Trends and Theory, 3-20.

Spielberger, C. D., y Díaz-Guerrero, R. (1975). Inventario de ansiedad: rasgo-estado. Manual Moderno.

Spielberger, C. D., Gorsuch, R. L., y Lushene, R. E. (1970). State-trait anxiety inventory manual. Mind Garden, Inc.

Spielberger, C. D., Gorsuch, R. L., Lushene, R. E., y Cubero, N. S. (1999). STAI: Cuestionario de ansiedad estado-rasgo. TEA ediciones.

Spielberger, C. D., Pollans, C. H., y Worden, T. J. (1984). Anxiety disorders. In M. Turner y M. Hersen (Eds.), Adult psychopathology and diagnosis (pp 263-303).

Tomás-Sábado, J. (2020). Miedo y ansiedad ante la muerte en el contexto de la pandemia de la COVID-19. Revista de Enfermería y Salud Mental, 16, 26-30. HTTP://DOI.ORG/10.5538/2385703X.2020.16.26

Vallejo Ruiloba, J. (1998). Introducción a la psicopatología y psiquiatría (4ta ed.). Masson.

Watzlawick, P., Beaving J. y Jackson, D. (1967). Teoría de la comunicación humana. Herder.

Wolpe, J. (1981). Reciprocal inhibition and therapeutic change. Journal of Behavior Therapy and Experimental Psychiatry, 12(3), 185-188.

Zavaleta, I. M. (2019). Un estudio sobre la percepción del empoderamiento femenino en redes sociales. Universidad de San Andrés. 